\title{
An Instructional Support Method for Students with Disabilities
}

\author{
Yxstian Gutierrez* \\ Department of Special Education, USA
}

*Corresponding author: Yxstian Gutierrez, Ed.D, Assistant Professor, Department of Special Education, USA.

Received Date: September 10, 2019

Published Date: September 20, 2019

\begin{abstract}
The purpose of this qualitative case study using grounded theory analysis was to explore the administration and implementation of shortened assignments for four students with disabilities and to describe the perceptions of co-teachers and parents of how shortened assignments assist or hinder their learning within a co-teaching context at an urban middle school. The Individual with Disabilities in Education Act (IDEA) 2009, a federal special education law, states that students with disabilities requiring special education services can use accommodations or adaptations of the curriculum to access the general education curriculum. A shortened assignment, as an accommodation, can help students with emotional disabilities learn and demonstrate their knowledge. Thorough interviews of these participants were conducted as well as classroom observations of how the shortened assignment was administered. Document analysis of student work samples was also conducted to explore how shortened assignments have been used with student class work. Data collected from the interviews, observations, and work samples answered four research questions.
\end{abstract}

\section{Introduction}

An IEP is a federal legal document developed and drafted by IEP team members that includes the parent, and addresses a student with a disability's educational setting, goals, progress, presentlevels, and accommodations/modifications [1]. Because of the provisions within the 2009 re-enactment of the Individuals with Disabilities in Education Act (IDEA, 2009), special education teachers are required to understand and implement the accommodations noted in their students with emotional disabilities' Individual Education Plans (IEPs) [2]. One typical accommodation listed in students' IEPs is shortened assignments. Periodically, teachers are not specifically taught how to implement this accommodation [3]. Teachers are often not provided insight into how to create an accommodation that will meet the needs of the student as specified in the IEP [4]. The federal law does not provide a definition of shortened assignments and how much is considered shortened. The word shortened is quite complex to understand since some students may have specific assignments reduced as determined by the IEP, while other students may have all assignments shortened. However, the federal law does state that the IEP team must have the discussion about how the shortened assignments will be administered in the classroom; for example, will it be an accommodation or modification (IDEA, 2009)? Will the teachers be able to reduce all assignments, and will all assignments be reduced? Who will administer this accommodation as well as determine the extent of how many questions or problems to reduce? According to IDEA, an accommodation only shortens the assignment, but allows the student to be exposed to the same concepts as a general education student; whereas, a "modification" alters the assignment to the point that the special education student is provided with different materials and curriculum from a general education student $[1,5]$.

\section{Background}

The nature of shortened assignments is quite complex since the accommodation has been used and can be applied in several ways. Until recently, some IEP teams with the cooperation of the case manager, also known as the special education teacher who is responsible for providing services, listed this accommodation on a student's IEP because the student had a learning disability [6]. Some general education teachers prefer to grade shortened assignments using the criteria of non-disabled students as opposed to using criteria stated in the IEP. Legally, special education and general education teachers must apply shortened assignments as an accommodation when a student has it listed on his or her IEP $[6,1]$. If the IEP team does not tie the accommodation to a subject or explain the extent of the accommodation, the teachers are then required to administer the accommodation in all classes. The IEP team is the only entity that can describe shortened assignments 
as an accommodation within the IEP [1]. The IEP states how the shortened assignments will be applied and the extent of the accommodation. Accommodations are often explained in the IEP with vague terms that results in teachers implementing the accommodation with inconsistency and inappropriately [7].

The co-teaching model can assist educators in implementing and following the IEP [8]. A co-teaching model is a way to collaborate with general education teachers in providing accommodations to students with disabilities. It is the act of teaching collaboratively with the general education teacher. Studies about co-teaching and instructional strategies to assist co-teachers in providing effective accommodations, including shortened assignments, have shown that the special education teacher education teacher will more likely ensure accommodations and IEP goals are being practiced $[10,11]$ and, that both teachers share ideas and learn from each other how to administer shortened assignments or other accommodations [6].

\section{Statement of the problem}

Teachers and parents often have difficulty understanding and implementing shortened assignments successfully [11,9]. Some teachers and administrators believe shortened assignments are too subjective and sometimes administered inconsistently. The perceptions of the co-teachers may affect how accommodations are provided since a co-teacher with a negative attitude about special education and not open to innovative methodologies may not be inclined to administer shortened assignments appropriately [4]. Researchers have documented such practices as teachers grading shortened assignments with a stricter grading scale [7,11,12]. Teachers have complained that shortened assignments give students preferential treatment.

Marshall Middle School (MMS- a pseudonym) is an urban middle school in which teachers have had difficulties with understanding and implementing shortened assignments. Similar to other researchers' findings, co-teachers have struggled to determine when to provide shortened assignments, and they have also experienced difficulty in agreeing how to grade such assignments $[3,13]$. No research has been conducted exploring how and when assignments are shortened for students with learning disabilities [5,7]. Research studies have been completed on collaborative models and the use of accommodations [14], but not with the use of shortened assignments for students with learning disabilities. Without significant improvement in administering this accommodation, the federal government may cite schools for not implementing IDEA effectively. Additionally, students may suffer from not having appropriate accommodations which may aid in their learning and help them avoid failing courses through experiencing difficulty in completing assignments.

\section{Purpose of the study}

The purpose of this qualitative case study was to explore the administration and implementation of shortened assignments for four students with learning disabilities and to describe the perceptions of co-teachers and parents about how shortened assignments benefit or hinder learning for these students within a co-teaching context at Marshall Middle School (MMS). This study may help improve both communication between co-teachers, and implementation of shortened assignments to best meet the needs of students with IEPs. The researcher explored the implementation and perceptions of the parents and co-teachers towards shortened assignments as an accommodation. Researching through a case study allowed the researcher to conduct an in-depth description, analysis, and interpretation of how this accommodation was implemented and understood through interviews with stakeholders (parents and co-teachers) and through observations of co-teaching contexts and document analysis of documents related to the teaching context, such as the students' IEPs (Yin, 2009). Using a case study method, recommendations illuminated the perceptions of participants about shortened assignments and possible ways to implement more effectively by examining the complex nuances involved in the creation and implementation of shortened assignments for these diverse students (Stake, 2009). The case study provided insight to current and future teachers on the success and challenges of providing this accommodation to students with disabilities in a coteaching setting.

\section{Research Questions}

The researcher conducted a transcription of thorough semistructured interviews and the implementation of a variety of qualitative grounded theory methodology techniques, such as the application of observations and student work samples. The researcher interviewed parents of four students with emotional disabilities (Appendix A). All co-teachers, including 2 General Education Language Arts Teachers (teachers A and B), 1 Special Education Teacher (teacher C), and 1 general education Math teacher (teacher D) were interviewed to examine their perceptions of the effectiveness of shortened assignments (Appendix B). The researcher also sought information by observing the co-teachers as they administered and implemented the accommodation (Appendix C). To triangulate the findings further, the researcher also used work samples to examine how shortened assignments were implemented and their effectiveness with students' grades within a co-teaching context. Member checking of the accuracy of what was transcribed was implemented as well as audio recordings of the interviews so that the researcher could review for accuracy and thoroughness. In addition, analyses of semi-structured interviews, observations, and work samples lead to four central themes which were grounded from the data: types of shortened assignments, lack of time/training, benefits to shortened assignments, and hindrance to shortened assignments. The following are the four research questions that guided this study:

Q1: How does the co-teaching model impact the implementation of shortened assignments?

Sub question. How do co-teachers understand the meaning of shortened assignments, so as to create effective assignments?

Sub question. How do co-teachers communicate with each other about this accommodation? 
Sub question. How do co-teachers work together to create and implement these accommodations?

Sub question. How do implemented shortened assignments reflect teachers' stated understandings of this accommodation?

Q2: How do parents understand the meaning of shortened assignments?

Q3: What are the co-teachers' perceptions of the effectiveness of this accommodation?

Q4: What are the parents' perceptions of the effectiveness of this accommodation?

\section{Methods}

This study examined the perceptions and experiences of stakeholders when administering or implementing shortened assignments as well as reviewed the factors that may either hinder or promote the accommodation to students with disabilities. The four research questions were addressed through methods of data collection, including interviews, observations, and artifacts. Using the case study method allowed the researcher to provide a thorough "story" of the individuals' experiences or perceptions [15]. The method allowed the researcher to focus only on the central questions, the experiences and perceptions of the coteachers and parents. In general, the case study method allows researchers to examine anything that is specific or unique and adds meaning to a study likewise, this study reviewed perceptions of parents and co-teachers which were meaningful to understanding shortened assignments. The case study provided insight to current and future teachers on the success and challenges of providing this accommodation to students with disabilities in a co-teaching setting.

Semi-structured interviews from faculty and parents were conducted to determine their input about the meaning and effectiveness of shortened assignments in a co-teaching model. Various in-depth interviews were held along with an analysis and interpretation of participants' responses to determine themes or patterns of their perceptions. All data was compared with observation and artifact data about shortened assignments. By completing this study, insights were gained about shortened assignments and how the accommodation was helpful or not to four students with emotional disabilities in a co-teaching setting. Special education psychological reports for each student were used as an artifact for this case study. These reports allowed the researcher to identify the students that had shortened assignments and gain insight to each student's educational and psychological histories. The researcher used several strategies to protect participants from any harmful effects, including the use of consent forms and audio recordings. The researcher will not publish the recordings. IRB approval was sought and received prior to any data collected.

\section{Population}

The process of recruitment of the parents and co-teachers began by the researcher considering a total of 290 students with a learning disability as classified in the student's IEP (Marshall Middle School, 2012). The initial number of eligible participants was narrowed to 45 students because they were enrolled in 7 th grade general education classes for the majority of the day. The researcher applied stratification or specific characteristics to narrow the results by searching only students who also received shortened assignments as an accommodation and a learning disability. This was completed by using the district's special education database website and by clicking on a filter box that narrowed student names by accommodations. The result of eligible students was 9 .

As a result of Marshall Middle School having a population of students of diverse Hispanic descent (94\%) and having students from low socio-economic (SES) backgrounds, the group included students of Hispanic descent, as a convenience that came from limited SES families. The California Department of Education (CDE) considers students who are eligible for "free and reduced" lunch to be considered low SES. This school site had $89 \%$ of students eligible for a free or reduced lunch.

\section{Sample}

This study included parents of four students with emotional disabilities and four co-teachers as its population. Although this study only considered four co-teachers and parents, five participants from each group were considered in case one of them decided not to participate in the study. However, no participants left the study. The co-teachers had at least three to five years full-time teaching experience and taught a class with learning disabled students who received shortened assignments. The four students had a diagnosis of emotional disturbance disability, were enrolled in the 7 th grade, and participated in the same Language Arts class within a coteaching setting. All four students had the same type of learning disability. In case one participant could no longer participate in the study, the researcher would have recruited a new participant who was enrolled in the same class as the other participants and enrolled in general education classes for the majority of the day.

The four students were in the seventh grade and participated in the Resource Specialist Program (RSP) which is considered a service option of special education; however, these students were in general education classes the majority of the day. The data was collected when the researcher interacted with the co-teachers in a classroom setting and the four parents at their homes. The four students received shortened assignments as part of their Individual Education Plan (IEP) which mandated this accommodation in all classes for all assignments. Shortened assignments were already part of these students' IEPs. Stratification or specific characteristics were used to identify eligible students. The selection of the four students was based on random selection of eligible students through Popsicle sticks. Each student that was eligible to participate in the study had their name written on a Popsicle stick and the primary investigator randomly selected four Popsicle sticks. The researcher continued this method of selecting participants, if original participants decided to not continue or choose to not participate. 
Stratification was applied in selecting the co-teachers and parents for this study. Only three general education teachers and one special education teacher who taught the selected students were selected. At least one general education teacher from Language Arts and Math participated. Only the parents of the selected students participated in this study. Once the researcher identified four eligible participants and one alternate, he contacted the parents to gain consent first through phone and then contacted the parents after school or at home to review and explain the consent form as well as obtain signatures prior to any data collection.

\section{Materials/Instruments}

This study used semi-structured interviews, observations, and document analysis as measurement tools [15]. The researcher administered semi-structured interviews. The interview questions along with the formatting were designed by the researcher (Appendix A). The interview protocol was field tested by three experts in special education from the school district. The school district's professional development specialist of special education field tested the protocol to ensure appropriate and accurate terminology and concepts. The protocol was then examined by the researcher's two colleagues who have taught for more than 15 years in special education and provided shortened assignments within a co-teaching setting. The researcher interviewed the four co-teachers and four parents to determine their perceptions of the administration and implementation of shortened assignments. Specifically, by using interview questions, the researcher addressed the perceptions of co-teachers and parents towards the meaning of shortened assignments. Different types of interview designs exist, including designs with predetermined answers, open ended questions, and informal conversations. This researcher designed the interviews with a standard open ended semi-structure in that the questions were selected by the researcher and the interviewees provided responses to open ended questions. Metaphorically, two types of interviewers exist: a traveler who works with the interviewee in discovering insights, and a miner who "unearths" data by continuing to ask questions. This researcher used the interview questions to discover the co-teachers and parents' perceptions of the effectiveness of shortened assignments which related to Klave's metaphor of an interviewer who is a "miner".

An observation protocol (Appendix B), designed by the researcher was also used. This protocol was also field tested by three experts in special education from the school district to ensure accuracy of the terminology and concepts. The researcher's experiences and job duties in administering shortened assignments for 6 years shaped the protocol design. The school district's professional development specialist of special education field tested the protocol as well as two colleagues with more than 15 years of experience administering shortened assignments within a coteaching setting. Observations of the co-teachers administering and providing the accommodations were conducted. Using observations and document analysis helped the researcher understand the nature of shortened assignments within a co-teaching setting as well as how shortened assignments are administered and implemented.

A document analysis of each student's IEP and work samples were examined to understand the complexities of shortened assignments and how they were used. The objective of observing the co-teachers and providing document analysis was to compare the data through the process of triangulation. Providing more than one perspective may strengthen the findings since the researcher may find consistent results [16]. Using more than one data source to answer the research questions may provide more credibility to the results. This type of triangulation involves the researcher using different types of sources to check the accuracy or certainty of the results.

Since this was a qualitative case study, thorough semistructured interviews were conducted by the researcher to understand co-teachers and parents' perceptions of shortened assignments. Interview questions were designed and implemented to see whether there was any insight to administering shortened assignments. All interview data was recorded and then transcribed onto a word document to help establish the trustworthiness of the data. The multiple interviews from co-teachers and parents helped the researcher to corroborate and triangulate the study's credibility. The structure and outline of the interview questions were the same for each participant. The researcher used separate participant interview sheets with standard questions and took separate extensive notes about each of the participants known as structured descriptions. All interview questions were designed by the researcher to seek in-depth knowledge of the research questions. Since the researcher for this study was bilingual, interview questions were asked in Spanish, to only Spanish speaking parents.

\section{Findings}

The results in the following table reflect findings from each research question. Table 1.

Table 1: Participants' Perceptions and Findings.

\begin{tabular}{|c|c|c|c|c|}
\hline Findings 1-4 & $\begin{array}{c}\text { Research Question 1 } \\
\text { Teachers' believed the } \\
\text { accommodation could be } \\
\text { used in many ways. }\end{array}$ & $\begin{array}{c}\text { Parents felt frustrated } \\
\text { when assignments are not } \\
\text { shortened. }\end{array}$ & $\begin{array}{c}\text { Some teachers perceive it as } \\
\text { helping to meet the student's } \\
\text { need for academic and behavior } \\
\text { purposes. }\end{array}$ \\
\hline Findings 5-8 & $\begin{array}{c}\text { Teachers did not } \\
\text { appropriately dminister the } \\
\text { accommodation due to lack } \\
\text { of training. }\end{array}$ & $\begin{array}{c}\text { Parents' lack of training was } \\
\text { a disadvantage in helping } \\
\text { teachers to implement the } \\
\text { accommodation effectively. }\end{array}$ & $\begin{array}{c}\text { General education teachers } \\
\text { perceive the accommodation as } \\
\text { short-term use. They perceive the } \\
\text { accommodation as helpful, but at } \\
\text { the same time limiting learning. }\end{array}$ \\
\hline
\end{tabular}




\begin{tabular}{|c|c|c|c|c|}
\hline Findings 9-12 & $\begin{array}{c}\text { Teachers' did not have } \\
\text { common prep periods to } \\
\text { plan and collaborate. }\end{array}$ & $\begin{array}{c}\text { Parents perceived it as } \\
\text { helping their children } \\
\text { receive not only better } \\
\text { grades, but also a reduction } \\
\text { in their child's frustration } \\
\text { level. }\end{array}$ & $\begin{array}{c}\text { The students' Special Education } \\
\text { teachers perceived the } \\
\text { accommodation as helpful. } \\
\text { Teachers viewed it as short-term } \\
\text { and long-term use. }\end{array}$ & $\begin{array}{c}\text { The accommodation should } \\
\text { be used for long-term use. }\end{array}$ \\
\hline
\end{tabular}

The data also generated four overlapping themes: a) types of shortened assignments; b) lack of time/training with shortened assignments; c) hindrance to learning; and d) benefits to learning. Specifically, the findings of this study revealed: a) teachers need more training in the administration and implementation of the accommodation; b) parents and teachers would benefit from dialogue about how the accommodation should be implemented; and c) participants perceived shortened assignments as beneficial, yet also enabling in a variety of ways. Data suggested that teachers and parents need a strong training program to fully understand the administration and implementation of shortened assignments as well as its effects in hindering or benefiting student learning.

\section{Recommendations}

As more students are being identified as students with special needs and receiving accommodations in the general education classroom, it is imperative that teachers and administrators, with the help of the parents, consider the following practical recommendations. Table 2 provides an overview of key recommendations:

Table 2: Key Recommendations to Emerging Issues/Themes.

\begin{tabular}{|c|c|c|c|c|}
\hline Emerging Themes/ Issues & Recommend \#1 & Recommend \#2 & Recommend \#3 \\
\hline Lack of Training/ Planning & $\begin{array}{c}\text { Ensure parents understand } \\
\text { the accommodation and } \\
\text { how it is implemented. }\end{array}$ & $\begin{array}{c}\text { Provide regular quarterly } \\
\text { trainings on-site and } \\
\text { constant classroom } \\
\text { visitations. }\end{array}$ & $\begin{array}{c}\text { School site administration } \\
\text { to allow common prep } \\
\text { periods between co- } \\
\text { teachers. }\end{array}$ & $\begin{array}{c}\text { Provide more hands-on } \\
\text { practical learning on how to } \\
\text { implement accommodations } \\
\text { in credentialing programs. }\end{array}$ \\
\hline Hindrance to Learning & $\begin{array}{c}\text { Discuss benefits and } \\
\text { negatives of accommodation } \\
\text { at IEP meetings. }\end{array}$ & $\begin{array}{c}\text { Grading procedures should } \\
\text { be established beforehand. }\end{array}$ & $\begin{array}{c}\text { first if they need the } \\
\text { accommodation. Ensure } \\
\text { skills from each standard } \\
\text { are administered. }\end{array}$ \\
$\begin{array}{c}\text { Both co-teachers should } \\
\text { be responsible in the } \\
\text { implementation. }\end{array}$ \\
\hline
\end{tabular}

To begin with, more on-site special education training to co-teachers in how to administer and implement shortened assignments within a co-teaching context needs to be implemented. Analysis of co-teacher interviews and classroom observations pointed out that co-teachers were not fully trained and experienced in administering and implementing shortened assignments. Data from the parents and co-teachers interviews and observations indicated inconsistencies in providing this accommodation. The school site could request the Special Education District Office to fund trainings for co-teachers at least in the beginning of the school year. The site administration should require co-teachers to attend and participate in a co-teacher training that includes practice and hands-on training in administering and implementing shortened assignments. Additionally, it may be beneficial to incorporate hands-on learning rather than lecture instruction in administering and implementing shortened assignments at these trainings as well as in teacher credential programs.

A second recommendation is for administrators and parents to provide more visible accountability and support for the co-teachers to ensure proper administration and implementation of shortened assignments. Classroom visitations have been effective tools to ensure teachers are teaching the standards and maintaining a learning environment [17]. At the same time, these researchers have agreed that the visitations to check for proper administration and implementation of shortened assignments can be beneficial in that they can serve as friendly reminders about best practices of using shortened assignments.
A third recommendation is for co-teachers or the student's case manager to provide parents with an overview of their son or daughter's accommodation, such as shortened assignments and how it will be administered and implemented. Although parents agree at IEP meetings that their son/daughter will receive shortened assignments, it is necessary for educators to providing this notification at the beginning of the school year to alert the parent of the accommodation being implemented as well as continue on-going communication. Parents who are aware of the special education process, shortened assignments, and accommodations can help ensure the student's IEP is being followed.

Additionally, it is recommended to the teachers and parents to allow the four students to decide prior to assignments being shortened whether they need the accommodation for that activity. The reasoning to allow student decision making is because the students may be able to complete the assignments entirely without the accommodation. If they can, the additional practice of class work may result in the student learning [3]. Providing shortened assignments without asking the students or determining if the student is able to complete the assignment may result in enabling behavior [18]. This is where the student can actually complete the assignment without any negative effects, such as feelings of frustration, but relies on the assignment being shortened.

The fourth recommendation is for teachers, parents, and administrators to ensure that co-teachers or the IEP team discusses shortened assignments as an accommodation during the IEP meeting with the parent and have a discussion at the IEP meeting 
and documented in the IEP how shortened assignments will be administered and implemented. For example, the IEP team needs to discuss and decide who will administer the accommodation and what assignments for what subjects will be shortened. The team also should discuss any grading concerns as a result of implementing shortened assignments. Data from parent interviews indicated that they did not fully understand how shortened assignments were graded. Co-teachers should regularly discuss the benefits or negatives of using shortened assignments at the IEP meeting and not arbitrarily "prescribe" shortened assignments for the next following school year. Parents should also initiate this conversation.

Another recommendation which was evident in the data is for administrators to continue to group students with this accommodation in common general education classrooms to make the administration and implementation of the accommodation more feasible for the co-teachers. For example, co-teachers may have an easier time implementing the accommodation if the groups of students who receive the accommodation are in the same class. This was evident in the case study, and it is recommended to keep doing this practice.

The final recommendation is for the school site administration to allow co-teachers to have the same prep period to discuss and plan how to implement shortened assignments for their selective students. Having a common prep period may allow the co-teachers to decide how shortened assignments will be graded as well as open a channel of communication as to how effective the accommodation is for the student.

\section{Future research}

One area of future research is to conduct a similar study but examine the perceptions of special education teachers who have students in self-contained classrooms. This dissertation study only examined co-teachers and parents' perceptions of how shortened assignments were implemented towards learning disabled students in the general education environment. Some students with special needs are educated in a separate classroom with only special education students because of their more severe academic, behavioral, or social needs. Another area of future interest is to conduct a quantitative study examining the effects of grading practices in applying shortened assignments. A survey or questionnaire with a Likert scale can be developed to examine how teachers grade shortened assignments and what criteria they used across several schools, if any.

Additionally, researchers may be interested to examine high school student perceptions of the administration and implementation of shortened assignments. Exploring the perceptions of high school students may be more feasible since students at that age level are more mature and can comprehend questions better than middle school students. It would also be interesting to see how older, more mature students view shortened assignments. For example, do these students see this accommodation as embarrassing in a general education classroom or do they perceive the accommodation as helpful in their learning?
Conducting a multiple case study of how teachers from several elementary and middle schools perceive shortened assignments may also contribute to the field of special education. The multiple case study may corroborate some of the findings from this study. It would be interesting to see what the elementary teachers' perceptions of how shortened assignments are administered and implemented; most elementary teachers have more teaching experience than middle and high school teachers. Elementary teachers spend most of the day with a group of students, so a researcher's findings about these teachers' lived experiences may be worthwhile.

\section{Conclusion}

The intention of this qualitative case study was to explore the administration and implementation of shortened assignments for four students with emotional disabilities and to describe the perceptions of co-teachers and parents about how shortened assignments benefited or hindered learning for these students within a co-teaching context at Marshall Middle School (MMS). This study helped improve both communication between coteachers, and implementation of shortened assignments to best meet the needs of students with IEPs. The researcher explored the implementation and perceptions of the parents and co-teachers towards shortened assignments as an accommodation. Researching through a case study allowed the researcher to conduct an in-depth description, analysis, and interpretation of how this accommodation was implemented and understood through interviews with stakeholders (parents and co-teachers) and through observations of co-teaching contexts and document analysis of documents related to the teaching context, such as the students' IEPs. Using a case study method, recommendations illuminate the perceptions of participants about shortened assignments and possible ways to implement more effectively by examining the complex nuances involved in the creation and implementation of shortened assignments for these diverse students [15]. The case study provided insight to current and future teachers on the success and challenges of providing this accommodation to students with disabilities in a co-teaching setting.

Data collected from the interviews, observations, and work samples answered the four research questions. The data also generated four overlapping themes:

a) types of shortened assignments

b) lack of time/training with shortened assignments

c) hindrance to learning and

d) benefits to learning.

The findings of this study demonstrated co-teachers and parents' perceptions of the administration and implementation of shortened assignments. Specifically, the findings of this study revealed:

a) teachers need more training in the administration and implementation of the accommodation 
b) parents and teachers would benefit with dialogue about how the accommodation should be implemented

c) participants perceived shortened assignments as beneficial, yet also enabling in a variety of ways [19].

The findings from this study contribute to the literature by providing co-teachers, parents, and administrators a better understanding of the perceptions of how shortened assignments were administrated and implemented within the context of this case study at Johnson Middle School. The findings provide additional insight to barriers co-teachers and parents face; as well as how they perceive the accommodation as benefiting or hindering learning within the context of a co-teaching setting. Data suggests that a teachers and parents need a strong training program to fully understand the administration and implementation of shortened assignments as well as its effects in hindering or benefiting student learning [20].

\section{Acknowledgement}

None.

\section{Conflict of Interest}

Author declare no conflict of interest.

\section{References}

1. US Department of Education (2018) Building the legacy: IDEA 2009. USA.

2. Hurd E, Weilbacher G (2017) You Want Me to Do What? The Benefits of Co-Teaching in the Middle Level. Middle Grades Review 3(1): 15.

3. Ketterlin Geller LR, Alonzo J, Braun Monegan J (2007) Recommendations for accommodations: Implications of inconsistency. Remedial and Special Education 28(4): 194-206.

4. Jackson K, Willis K, Giles L, Lastrapes R, Mooney P (2017) How to Meaningfully Incorporate Co-teaching Into Programs for Middle School Students With Emotional and Behavioral Disorders. Beyond Behavior 26(1): 11-18.

5. Gurger, Uzuner (2010) A phenomenological analysis of the views on coteaching applications in the inclusion classroom. Educational Sciences: Theory and Practice 10(1): 311-331.

6. Christle CA, Yell ML (2010) Individualized education programs: Legal requirements and research findings. Exceptionality 18(3): 109-123.
7. Worrell J (2008) How secondary schools can avoid the seven deadly school "sins" of inclusion. American Secondary Education 36(2): 43-56.

8. Carty A, Marie Farrell A (2018) Co-teaching in a mainstream postprimary mathematics classroom: an evaluation of models of co-teaching from the perspective of the teachers. Support for Learning 33(2): 101121.

9. Preves S, Stephenson D (2014) The classroom as stage: Impression management in collaborative teaching. Teaching Sociology 37(3): 245256.

10. Pugach M, Winn J (2011) Research on co-teaching and teaming. Journal of Special Education Leadership 24(1): 36-46.

11. O Rourke J, Houghton S (2015) Perceptions of secondary school students with mild disabilities to the academic and social support mechanisms implemented in regular classrooms. International Journal of Disability, Development \& Education 55(3): 227-237.

12. Suk Hyang L, Michael L Wehmeyer, Jane H Soukup, Susan B Palmer (2010) Impact of curriculum modifications on access to the general education curriculum for students with disabilities. Exceptional Children 76(2): 213-233.

13. Obiakor FE, Harris M, Mutua K, Rotatori A, Algozzine B (2012) Making inclusion work in general education classrooms. Education \& Treatment of Children (West Virginia University Press) 35(3): 477-490.

14. Akçamete G, Dagli Gokbulut O (2018) Opinions of classroom teachers on reading comprehension difficulties, inclusion education and coteaching. Quality \& Quantity 52(1): 791-806.

15. Stake R E (2009) The incredible lightness of evidence: Problems of synthesis in educational evaluation. Studies in Educational Evaluation 35: 3-6

16. Erwin E J, Brotherson MJ, Summers JA (2011) Understanding qualitative metasynthesis: Issues and opportunities in early childhood intervention research. Journal of Early Intervention 33(3): 186-200.

17. Simmons RJ, Magiera K (2017) Evaluation of co-teaching in three high schools within one school district: How do you know when you are truly co-teaching? Teaching Exceptional Children Plus 3(3): 1.

18. Elbaum B (2007) Effects of an oral accommodation on the mathematics performance of secondary students with and without learning disabilities. The Journal of Special Education 40(4): 218-229.

19. Nguyen H (2012) General education and special education teachers collaborate to support English language learners with learning disabilities. Issues in Teacher Education 21(1): 127-152.

20. Wassell B, Lavan S (2009) Tough transitions? Mediating beginning urban teachers' practices through coteaching. Cultural Studies of Science Education 4(2): 409-432. 